\title{
Practical Applicability of Teledentistry in Pediatric Patients Amidst Pandemic : A Narrative Review
}

\author{
Mousumi Goswami ${ }^{1 *}$, Tanu Nangia ${ }^{1}$, Aditya Saxena ${ }^{1}$, Sakshi Chawla ${ }^{1}$, Anam Mushtaq ${ }^{1}$, \\ Shrey R. Singh ${ }^{2}$ and Palak Jain ${ }^{1}$ \\ ${ }^{1}$ I.T.S Dental College, Hospital and Research Centre, Noida, India, ${ }^{2}$ School of Medical Science and Research, Sharda \\ University, Noida, India
}

\section{OPEN ACCESS}

Edited by:

Ola B. Al-Batayneh,

Jordan University of Science and

Technology, Jordan

Reviewed by:

Ahmad Faisal Ismail,

International Islamic University

Malaysia, Malaysia

Arlette Setiawan

Universitas Padjadjaran, Indonesia

*Correspondence:

Mousumi Goswam

mousumi87goswami@gmail.com

Specialty section:

This article was submitted to

Pediatric Dentistry,

a section of the journal

Frontiers in Dental Medicine

Received: 27 July 2021 Accepted: 21 September 2021

Published: 27 October 2021

Citation:

Goswami M, Nangia T, Saxena A, Chawla S, Mushtaq A, Singh SR and Jain $P$ (2021) Practical Applicability of

Teledentistry in Pediatric Patients

Amidst Pandemic : A Narrative

Review. Front. Dent. Med. 2:748089.

doi: 10.3389/fdmed.2021.748089
The coronavirus disease-2019 (COVID-19) pandemic has imposed a situation where all healthcare facilities except emergency services remain suspended. These times generated the necessity for the implementation of a healthcare delivery system that can be accessed digitally and, thus, benefit the majority of children as well as healthcare professionals. This review aims to propose a sound model of less technique sensitive, safe and handy strategies for dental traumatic injuries, endodontic and restorative concerns, and orthodontic urgencies until complete clinical help can be sought. Five hundred thirty articles were obtained from the PubMed, Google Scholar, Embase, Lilacs, and Cochrane databases published from 2011 to 2021. Nineteen articles that described teledentistry in the COVID-19 era were included. Teledentistry can serve as a vital patient management strategy that can aid in triaging urgent and elective patient treatment needs, ultimately easing the burden of clinics and at the same time providing a safer means of consultation.

Keywords: teledentistry, telehealth, teleconsultation, pediatric dentistry, COVID-19

\section{INTRODUCTION}

Dentistry has been considered as the most susceptible profession to get affected by the COVID19 pandemic, as it involves close inspection, examination, diagnosis, and therapeutic interventions around the naso-oropharyngeal region (1). This enormously aggressive novel coronavirus SARS CoV-2, whose epicenter was Hubei province in China, was declared as a health emergency of global concern by the World Health Organization (WHO) on January 30, 2020 (2). Around the world, even after 1 year, people are still experiencing suspension of ongoing dental practices except for addressing emergent needs (3-5). A rising number of pediatric cases of COVID-19 infection with greater severity and higher risk of transmission have been seen in the second wave of the pandemic $(6,7)$. This has made it imperative for pediatric dentists to be vigilant in triaging, assessing, and managing patients.

During these difficult times, adapting a telehealth model has been the most sound approach practiced by all nations to manage patients presenting with health problems during lockdown and otherwise. Advisories also focus on managing the majority of patients affected by COVID19 within the home under the supervision of a treating doctor via telehealth (8). In dentistry, this approach has been proven to be successful, as it satisfies social distancing norms and allows halting the transmission of the virus to contain its spread. 
Teledentistry has been defined by Cook in 1997 as "the practice of using video-conferencing technologies to diagnose and advice about treatment over a distance" $(9,10)$. Teledentistry, a form of telehealth comprising of a synergism between telecommunications and dentistry that involves the exchange of clinical derived information and relevant images for consultation and treatment planning.

The concept has proven to be a boon for the continuous delivery of necessary healthcare services during difficult situations of the pandemic (11). Innovations in the form of mobile applications and other technical developments have eased the learning and referral protocol necessary to minimize direct contact between patients and doctors.

\section{METHODOLOGY}

An electronic search from databases of PubMed, Google Scholar, Embase, Lilacs, and Cochrane was conducted for articles published from 2011 to June 2021. The use of MeSH terms "teledentistry", "teledentistry" AND "COVID-19" yielded a total of 530 articles (Pubmed: 256, Google scholar: 270, and Embase via Cochrane: 2) out of which 211 duplicates and 5 non-English publications were removed. Screening of the remaining 314 articles was performed based on title and abstract, and the articles were sought for retrieval. Full texts of only 147 articles were retrieved and screened for further eligibility. Articles published as reviews, systematic reviews, meta-analysis, observational studies, and randomized clinical trials were included while those published as case reports, books or documents, and conference proceedings were excluded. The exclusion was also made after screening based on relevance to dentistry, pediatric or general dentistry, triaging of patients, guidelines, protocol, and reviews from clinical experience. Out of the 147 articles, 19 that described a practical implementation of teledentistry service in the COVID19 era were selected for the review $(3-5,8,11-25)$. Refer to the flowchart in Supplementary Figure 1.

\section{Types of Teledentistry}

1. Two-Way Interactive or Real-Time Consultation/ Synchronous

Real-time consultation caters to video conferencing between a dentist and a patient with simultaneous exchange of information, medical history, and reports in order to reach a diagnosis within the same appointment. It also aids in the prompt exchange of information and reporting with a peer dentist or specialist. Two- way interactive technology allows procuring live images or sound occurring in an originating site to a person in a remote or distant site (26-31).

2. Store and Forward Teledentistry/Asynchronous

Information is gathered from one location, stored, and transferred to the treating consultant in a different location. Relevant data in the form of x-rays, photographs, and scanned images are uploaded and forwarded to the consultant after screening or storing it in the system.

This system is beneficial for consulting a specialist/team from a different place/city/country before referring or managing the patient. The data can be used to educate colleagues after obtaining a prior consent. A pattern of asynchronous consultation is also available on many webpages for fetching a potential diagnosis by entering serology or pathology reports (30, 31).

\section{Remote Monitoring of Patient}

Information on health and other medical data are transmitted from one location to another via electronic media for healthcare and supportive management.

\section{Mobile Health (mHealth)}

The use of mobile communication devices to support public health practice and education by using devices such as cell phones, tablets, computers, and personal digital assistants (PDAs).

\section{Telehealthcare Model Teletriaging}

Categorizing patients based on the urgency of treatment can be managed by teletriage. The dental team or front office can characterize if the case of a patient is elective or emergent. Emergent cases can be prioritized, and elective cases can be scheduled at a convenient time for teleconsultation, and appointments can be postponed to a more appropriate time (12). "Forward triage" helps to reduce the workload of a caregiver by managing non-emergent patients before they visit a hospital (32, 33). It foresees any crisis that can occur and prevents unnecessary movements in times of pandemic.

\section{Teleconsultation}

Interactive consultation with a clinician by telephonic or video conferencing is the prime component of the telehealth model. Consultation with a specialist can also be included if the software allows multiple participants at a time. Exchange of chief complaint, medical history, recent and previous laboratory reports, extraoral photographs, intraoral photographs, dental cast photographs, and radiographs, and examination by virtual appointments comprises a teleconsultation.

\section{Telediagnosis}

Analysis of collected information in teleconsultation leads to formulation of a diagnosis. The appropriate diagnosis and devised treatment plan are projected to the patient. Efforts were made in the past by Haron et al. to develop "Mobile Mouth Screening Anywhere (MeMoSA $\AA)$ )" for detection of oral cancer (34). Limited access to specialist has also lead to the development of tablet based mobile microscope (Cellscope Device) as an adjust to oral cancer screening (35).

\section{Telemonitoring}

Follow-ups and routine checkups in dentistry have always been of less priority among patients. Monitoring of postoperative cases with the help of telecommunication can be effectively carried out. Taking the help from scheduled telephonic calls, video conferencing, or merely by filling e-forms about resolution of symptoms can pave the way for sound dental health and can anticipate any treatment failure to occur (36). 


\section{Applicability in Pediatric Dentistry}

The American Academy of Pediatric Dentistry (AAPD) recognizes COVID-19 as an ongoing community and global problem with ever increasing number of cases among children and have, thereby, added a COVID-19 resource page in the recent reference manual, AAPD 2020-2021 namely "Re-emergence A Report on Pediatric Dental Practice Re-entry into Practice During the COVID-19 Pandemic" (13), also holds the statement for parent-FAQ (37). It also highlights the implementation of public health initiatives and the impact on oral healthcare delivery during the suspension of dental practice.

Adoption of teledentistry in daily practice:

1. Conduction of regular virtual continuous dental education programs and webinars for dentists to appraise various recent software and technological platforms as a medium for practicing teledentistry.

2. Dissemination of approaches for adequate management by dentists can be delivered by hospital-based software with stepwise triaging of patients. The hospital-based software can also be converted into a self-help application with which patients can direct themselves according to their chief complaint and acquire help from a specialist by scheduling a call or initiating a conversation. Another approach of providing management options can be applicable in private dental practices where patients can talk to their treating doctors directly for help.

Pediatric patients may have a variety of treatment needs that requires definitive operative, endodontic, orthodontic, or surgical intervention in a dental clinic/hospital. We propose a model of teledentistry-assisted management of pediatric dental problems during the COVID-19 pandemic that can be augmented with video-based and live teleconsultation demonstrations in order to minimize the need for dental clinic visits. It includes at-home recommendations for non-emergency concerns that can be disseminated via teleconsultation mode for parents and caregivers.

The main aim of this approach is to provide help during the pandemic when patients are unable to seek definitive treatment at the professional level because of the risk of infection, overburdened hospitals, and unavailability of functioning dental offices during lockdowns. The proposed specific problemoriented management model is described in Tables 1, 2. These tables describe proposed advice and possible modified management based on established guidelines. It must not replace the definitive treatment required for a patient in a dental clinic that involves thorough clinical examination and adjunctive tests for accurate diagnosis and treatment plan (14, 38-47).

\section{Recent Evidence of Teledentistry}

As the dynamics of the pandemic are changing with time, the acquisition of updated knowledge of the usage and practical applications as well as challenges of teledentistry is important before designing any robust model of action. According to a survey by Dusseja et al. almost $70.7 \%$ of respondents were unwilling to visit dental clinics during the COVID-19 pandemic, and $80.5 \%$ of the participants were in favor of teledentistry to resolve their dental problems. Out of them, $58 \%$ of the responders received prescriptions via WhatsApp, 35\% through telephone, $5 \%$ through video call, and $2 \%$ via e-mail and other means (17). Similarly, in a study by Davies et al. findings show that over $50 \%$ of photographic referrals resulted in either advice or antibiotics being prescribed, without the need for face-to-face contact. Over $70 \%$ of the images received during the study period were related to children aged 10 and under, and the majority related to swelling, pain, and dental trauma (18). Furthermore, a survey by Statista reports that $97 \%$ of those between the ages of 16 and 54 yr now own a smartphone with the capability to take photographs and send email (48).

In a survey by Rahman et al., patient satisfaction was evaluated along with ease of use, effectivity in accessing clinical services, and reliability of the teledentistry system; $94 \%$ of the respondents were satisfied with telephonic consultation and willing to utilize it in the future. Majority of the respondents were able to express themselves satisfactorily and clearly (19). A recent study from Google interest trends on self-medication during the COVID19 pandemic depicted an increase in the number of searches regarding self-medication, which indicates the need to reach out to the population in different ways, one of which can be through teledentistry, as reported by Sen Tunc et al. (20).

A few more acceptance-oriented studies have achieved good responses with teledentistry consultation for pain as well as for monitoring and follow-ups. Teledentistry has been beneficial in various fields, such as orthodontics, maxillofacial surgery, and pediatric dentistry (21-23).

\section{Barriers in the Use of Teledentistry}

1. Technology: The complexity of technology might make it difficult for clinicians to accept teledentistry, as they may be hesitant to learn and adopt a new skill.

2. Practical implications: Inability to perform a tactile examination of lesion/oral cavity and two-dimensional pictures, and improper evaluation of interproximal contact and posterior-most teeth might offer a limitation in accurate diagnosis (24). Clinicians might get afraid of making a wrong diagnosis and inappropriate treatment plan.

3. Communication: Patient acceptance due to lack of in-person communication may hamper in adopting a devised treatment plan, although many surveys being conducted support the gaining popularity of teledentistry $(17-20,48)$.

4. Rural setting: Lack of infrastructure such as internet connectivity, computer or smartphone, $\mathrm{x}$-ray machine and other advance armamentarium to help in diagnosing the tooth/lesion, providing it to the patient or transferring to a specialist at different location (25).

5. Privacy: The lack of privacy of transferred information may be a roadblock in gaining the confidence of patients, although the law for breach in privacy of a patient without his/her consent is strict. It is advised to all healthcare workers and portals working for telehealth to obey the Health Insurance Portability and Accountability Act (HIPAA) ${ }^{1}$.

\footnotetext{
${ }^{1}$ Available online at: https://www.cdc.gov/phlp/publications/topic/hipaa.html.
} (accessed July 06, 2021). 
TABLE 1 | Management of common pediatric dental problems.

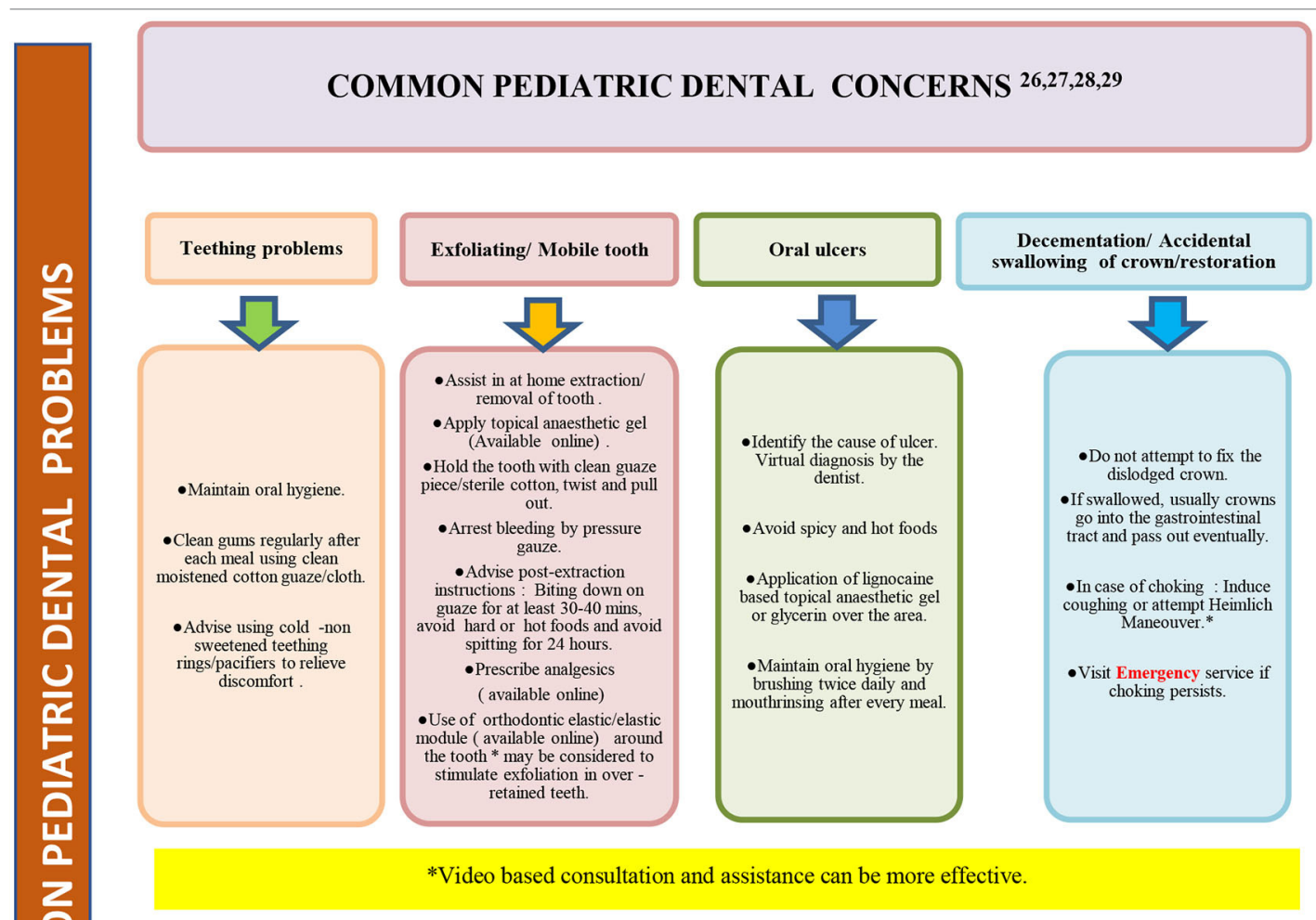

\section{CARIOUS LESION \& LESION INVOLVING PULP 30,31,32,33,34}

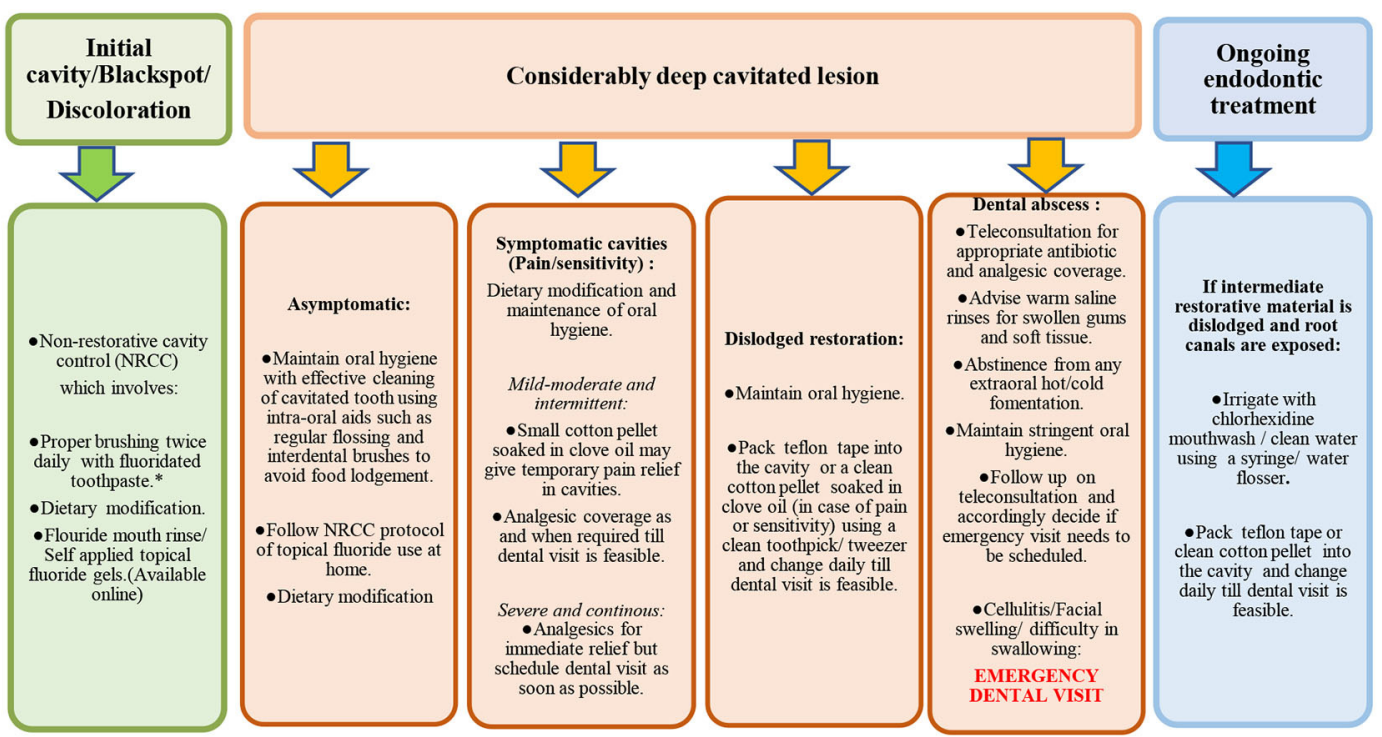

*Video based consultation, assistance and live demonstration for age appropriate tooth brushing can be more effective. 
TABLE 2 | Management of common pediatric dental problems.

\section{DENTAL TRAUMA ${ }^{35,36}$}
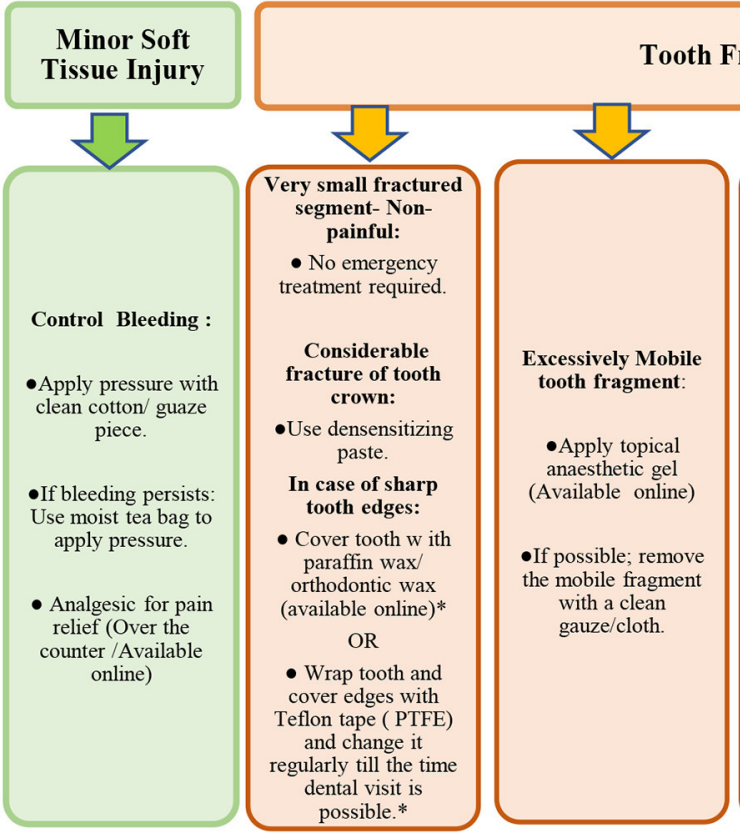

\section{ooth Fracture} maintenance of strict oral hygiene

\section{PEDIATRIC ORTHODONTICS ${ }^{37,38}$}

\section{Broken space maintainers / appliances}

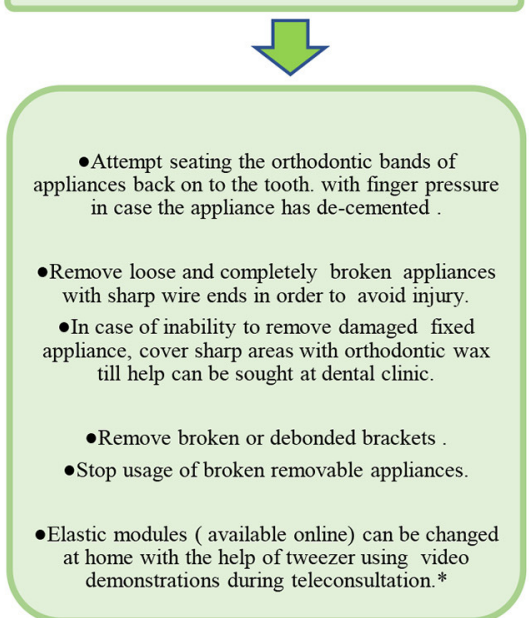

\section{Impinging wires and brackets}

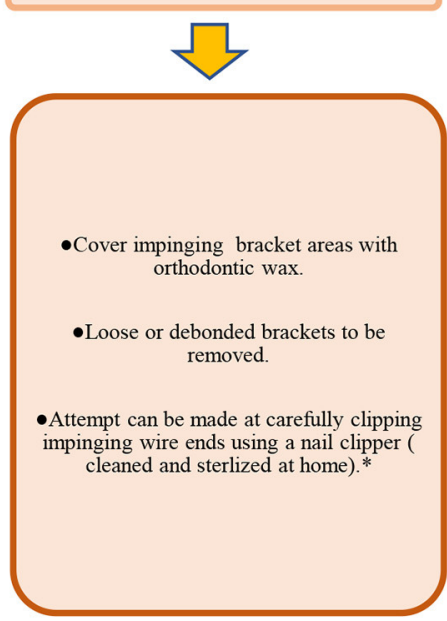

Accidental swallowing

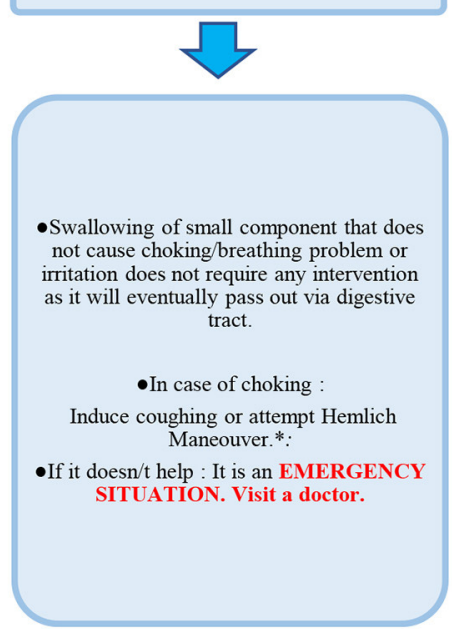

*Video based consultation, assistance and live demonstration for removal or adjusting appliance can be done. 


\section{SUMMARY}

Telemedicine has been widely used in the COVID-19 pandemic. Dentistry in the newer times does not only rely on technological advancements in terms of armamentarium and instruments but also ways to reach out to patients using convenient and advanced modes of communication media that include teleconsultation support anytime and anywhere as well as internet-based social media platforms for creating health awareness and disseminating valuable information to patients during times of crisis. Teledentistry can, therefore, serve as a vital patient management strategy that aids in triaging urgent and elective patient treatment needs, ultimately easing the burden of clinics and at the same time providing a safer means of consultation.

\section{REFERENCES}

1. Peng X, Xu X, Li Y, Cheng L, Zhou X, Ren B. Transmission routes of 2019-nCoV and controls in dental practice. Int J Oral Sci. (2020) 12:9. doi: 10.1038/s41368-020-0075-9

2. World Health Organization. Statement on the second meeting of the International Health Regulations (2005). Emergency Committee regarding the outbreak of novel coronavirus (2019-nCoV). Available online at: https:// www.who.int/news-room/detail/30-01-2020-statement-on-the-secondmeeting-of-the-international-health-regulations-(2005)-emergencycommittee-regarding-the-outbreak-of-novel-coronavirus-(2019-ncov) (accessed May 15, 2021).

3. Amato A, Caggiano M, Amato M, Moccia G, Capunzo M, De Caro F. Infection control in dental practice during the COVID-19 pandemic. Int $J$ Environ Res Public Health. (2020) 17:4769. doi: 10.3390/ijerph17134769

4. Duruk G, Gumusboga ZS, Colak C. Investigation of Turkish dentists' clinical attitudes and behaviors towards the COVID-19 pandemic: a survey study. Braz Oral Res. (2020) 34:e054. doi: 10.1590/1807-3107bor-2020.vol3 4.0054

5. Guo H, Zhou Y, Liu X, Tan J. The impact of the COVID-19 epidemic on the utilization of emergency dental services. J Dent Sci. (2020) 15:56467. doi: 10.1016/j.jds.2020.02.002

6. Iftimie S, López-Azcona AF, Vallverdú I, Hernández-Flix S, de Febrer G, Parra S, et al. First and second waves of coronavirus disease-19: a comparative study in hospitalized patients in Reus, Spain. PLoS ONE. (2021) 16:e248029. doi: 10.1101/2020.12.10.20246959

7. Brookman S, Cook J, Zucherman M, Broughton S, Harman K, Gupta A. Effect of the new SARS-CoV-2 variant B117 on children and young people. Lancet Child Adolesc Health. (2021) 5:9-10. doi: 10.1016/S2352-4642(21)00030-4

8. Monaghesh, E., Hajizadeh, A. The role of telehealth during COVID-19 outbreak: a systematic review based on current evidence. BMC Public Health. (2020) 20:1193. doi: 10.1186/s12889-020-09301-4

9. Fricton J, Chen $H$. Using teledentistry to improve access to dental care for the underserved. Dent Clin North Am. (2009) 53:537-48. doi: 10.1016/j.cden.2009.03.005

10. Cook J, ISDN. video conferencing in postgraduate dental education and orthodontic diagnosis. Learning Technology in Medical Education Conference. CTI Med. (1997) 1997:111-6.

11. Ghai S. Teledentistry during COVID-19 pandemic. Diabetes Metab Syndr. (2020) 14:933-35. doi: 10.1016/j.dsx.2020.06.029

12. Goswami, M. Chawla S. Time to restart: a comparative compilation of triage recommendations in dentistry during the Covid-19 pandemic. J Oral Biol Craniofac Res. (2020) 10:374-84. doi: 10.1016/j.jobcr.2020.06.014

13. American Academy of Pediatric Dentistry. Re-emergence Pediatric Dentistry Practice Checklist: A guide for re-entry into practice for pediatric dentists during the COVID-19 pandemic. Available online at: https://www.aapd.org/ link/8a3864bdb3684db19145486aca0d4bcc.aspx (accessed May 15, 2021).

\section{AUTHOR CONTRIBUTIONS}

MG, TN, AS, SC, AM, SS, and PJ organized database, wrote first draft, sections of the manuscript, conception, and design of the study. All authors contributed to the manuscript revision, read, and approved the submitted version.

\section{SUPPLEMENTARY MATERIAL}

The Supplementary Material for this article can be found online at: https://www.frontiersin.org/articles/10.3389/fdmed. 2021.748089/full\#supplementary-material
14. Martignon S, Cortes A, Douglas GV, Newton JT, Pitts NB, Avila V, et al. CariesCare International adapted for the pandemic in children: Caries OUT multicentre single-group interventional study protocol. BMC Oral Health. (2021) 21:329. doi: 10.1186/s12903-021-01674-1

15. Aziz SB, Kapoor P. Customized orthodontic triage during COVID-19 pandemic. J Indian Orthodontic Soc. (2020) 54:3913. doi: $10.1177 / 0301574220963495$

16. Luzzi V, Ierardo G, Bossù $M$, Polimeni A. Paediatric Oral Health during and after the COVID-19 Pandemic. Int J Paediatr Dent. (2021) 31:206. doi: 10.1111/ipd.12737

17. Dusseja SH, Rao D, Panwar S, Ameen S. Patients' views regarding dental concerns and tele dentistry during COVID-19 pandemic Int J Sci Healthc Res. (2020) 5:423-9. Available online at: https://ijshr.com/IJSHR_Vol.5_Issue.4_ Oct2020/IJSHR_Abstract.0056.html

18. Davies A, Howells R, Lee SMG, Sweet CJ, Dominguez-Gonzalez S. Implementation of photographic triage in a paediatric dental, orthodontic, and maxillofacial department during COVID-19. Int J Paediatr Dent. (2020) 31:547-53. doi: 10.1111/ipd.12773

19. Rahman N, Nathwani S, Kandiah T. Teledentistry from a patient perspective during the coronavirus pandemic. Br Dent J. (2020) 229:14. doi: 10.1038/s41415-020-1919-6

20. Sen Tunc E, Aksoy E, Arslan HN, Kaya Z. Evaluation of parents' knowledge, attitudes, and practices regarding self-medication for their children's dental problems during the COVID-19 pandemic: a crosssectional survey. BMC Oral Health. (2021) 21:98. doi: 10.1186/s12903-021-01 466-7

21. Saccomanno S, Quinzi V, Sarhan S, Laganà D, Marzo G. Perspectives of tele-orthodontics in the COVID-19 emergency and as a future tool in daily practice. Eur J Paediatr Dent. (2020) 21:157-62. doi: 10.23804/ejpd.2020.21.02.12

22. Menhadji P, Patel R, Asimakopoulou K, Quinn B, Khoshkhounejad G, Pasha $\mathrm{P}$ et al. Patients' and dentists' perceptions of Tele-Dentistry at the time of COVID-19. A questionnaire-based study: Tele-dentistry at the time of COVID-19. J Dent. (2021) 13:103782. doi: 10.1016/j.jdent.2021.103782

23. Machado RA, de Souza NL, Oliveira RM, Martelli Júnior H, Bonan PRF. Social media and telemedicine for oral diagnosis and counselling in the COVID-19 era. Oral Oncol. (2020) 105:104685. doi: 10.1016/j.oraloncology.2020.104685

24. Smith AC, Thomas E, Snoswell CL, Haydon H, Mehrotra A, Clemensen J, et al. Telehealth for global emergencies: implications for coronavirus disease 2019 (COVID-19). J Telemed Telecare. (2020) 26:309-13. doi: 10.1177/1357633X20916567

25. Nuvvula S, Mallineni SK. Remote management of dental problems in children during and post the COVID-19 pandemic outbreak: a teledentistry approach. Dent Med Probl. (2021) 58:237-41. doi: 10.17219/dmp/133182

26. Jampani ND, Nutalapati R, Dontula BS, Boyapati R. Applications of teledentistry: a literature review and update. J Int Soc Prev Community Dent. (2011) 1:37-44. doi: 10.4103/2231-0762.97695 
27. Clark GT. Teledentistry: What is it now, and what will it be tomorrow? J Calif Dent Assoc. (2000) 28:121-7.

28. Folke LE. Teledentistry. An overview. Tex Dent J. (2001) 118:10-8.

29. Farman AG, Farag AA. Teleradiology for dentistry. Dent Clin North Am. (1993) 37:669-81.

30. Bhambal A, Saxena S, Balsaraf SV. Teledentistry: potentials unexplored. J Int Oral Health. (2010) 2:1-6. doi: 10.1177/2229411220110105

31. Golder DT, Brennan KA. Practicing dentistry in the age of telemedicine. J Am Dent Assoc. (2000) 131:734-44. doi: 10.14219/jada.archive.2000.0272

32. Daniel SJ, Wu L, Kumar S. Teledentistry: a systematic review of clinical outcomes, utilization and costs. J Dent Hyg. (2013) 87:345-52.

33. Estai M, Kanagasingam Y, Tennant M, Bunt S. A systematic review of the research evidence for the benefits of teledentistry. J Telemed Telecare. (2018) 24:147-56. doi: 10.1177/1357633X16689433

34. Haron N, Zain RB, Ramanathan A, Abraham MT, Liew CS, Ng KG. mHealth for early detection of oral cancer in low- and middle-income countries. Telemed J e Health. (2020) 26:278-85. doi: 10.1089/tmj.2018.0285

35. Skandarajah A, Sunny SP, Gurpur P, Reber CD, D'Ambrosio MV, Raghavan N, et al. Mobile microscopy as a screening tool for oral cancer in India: a pilot study. PLoS ONE. (2017) 12:e0188440. doi: 10.1371/journal.pone.0188440

36. Mariño R, Ghanim A. Teledentistry: a systematic review of the literature. $J$ Telemed Telecare. (2013) 19:179-83. doi: 10.1177/1357633x13479704

37. American Academy of Pediatric Dentistry. Talking to Parents about the COVID-19 Pandemic. Available online at: https://www.aapd.org/globalassets/ media/covid-19/aapdparentfaq.pdf (accessed May 1, 2021).

38. Owais AI, Zawaideh F, Bataineh O. Challenging parents' myths regarding their children's teething. Int J Dent Hygiene. (2010) 8:28-34. doi: 10.1111/j.1601-5037.2009.00412.x

39. Regev E, Lustmann J, Nashef R. Atraumatic teeth extraction in bisphosphonate-treated patients. J Oral Maxillofac Surg. (2008) 66:1157-61. doi: 10.1016/j.joms.2008.01.059

40. Tarakji B, Gazal G, Al-Maweri SA, Azzeghaiby SN, Alaizari N. Guideline for the diagnosis and treatment of recurrent aphthous stomatitis for dental practitioners. J Int Oral Health. (2015) 7:74-80.

41. Vranić DN, Jurković J, Jeličić J, Balenović A, Stipančić G, Cuković-Bagić I. Medical Emergencies in Pediatric Dentistry. Acta Stomatol Croat. (2016) 50:72-80. doi: 10.15644/asc50/1/10

42. Fontana M. Nonrestorative management of cavitated and noncavitated caries lesions. Dental Clinics. (2019) 63:695-703. doi: 10.1016/j.cden.2019. 06.001
43. Olcay K, Steier L, Erdogan H. Polytetrafluoroetylene tape as temporary restorative material: a fluid filtration study. J IstanbUnivFac Dent. (2015) 49:17-22. doi: 10.17096/jiufd.08659

44. Azim AA, Shabbir J, Khurshid Z, Zafar MS, Ghabbani HM, Dummer PM. Clinical endodontic management during the COVID-19 pandemic: a literature review and clinical recommendations. Int Endod J. (2020) 53:146171. doi: $10.1111 /$ iej.13406

45. Jyoti BB. Phytotherapeutics in conservative dentistry \& endodontics -a review. J Conserv Dent. (2005) 8:31-9. doi: 10.4103/0972-0707.42602

46. Fouad AF, Abbott PV, Tsilingaridis G, Cohenca N, Lauridsen E, Bourguignon C, et al. International Association of Dental Traumatology guidelines for the management of traumatic dental injuries: 2 avulsion of permanent teeth. Dent Traumatol. (2020) 36:331-42. doi: 10.1111/edt.12573

47. Bourguignon C, Cohenca N, Lauridsen E, Flores MT, O’Connell A, Day PF, et al. International Association of Dental Traumatology guidelines for the management of traumatic dental injuries: 1 Fractures and luxations. Dent Traumatol. (2020) 36:314-30. doi: 10.1111/edt.12578

48. O'Dea S.Smartphone Ownership in the United Kingdom (UK). 20122020, by Age. 2020. Available online at: www.statista.com/statistics/271851/ smartphone-owners-in-the- united-kingdom-uk-by-age/. (accessed July 25, 2020).

Conflict of Interest: The authors declare that the research was conducted in the absence of any commercial or financial relationships that could be construed as a potential conflict of interest.

Publisher's Note: All claims expressed in this article are solely those of the authors and do not necessarily represent those of their affiliated organizations, or those of the publisher, the editors and the reviewers. Any product that may be evaluated in this article, or claim that may be made by its manufacturer, is not guaranteed or endorsed by the publisher.

Copyright (c) 2021 Goswami, Nangia, Saxena, Chawla, Mushtaq, Singh and Jain. This is an open-access article distributed under the terms of the Creative Commons Attribution License (CC BY). The use, distribution or reproduction in other forums is permitted, provided the original author(s) and the copyright owner(s) are credited and that the original publication in this journal is cited, in accordance with accepted academic practice. No use, distribution or reproduction is permitted which does not comply with these terms. 